\title{
Khat Associated End Stage Chronic Liver Disease: A Case Report
}

\author{
Imran M. Patanwala ${ }^{\mathrm{a}, \mathrm{b}, \mathrm{c}}$, Alastair D. Burt ${ }^{\mathrm{b}}$, Margaret F. Bassendine ${ }^{\mathrm{a}, \mathrm{b}}$, \\ Mark Hudson ${ }^{\mathrm{a}, \mathrm{b}}$
}

\begin{abstract}
Khat induced hepatitis and chronic liver disease is often an overlooked cause of liver related morbidity in immigrant populations from East Africa and Yemen. Medical and psychological effects of Khat abuse are well reported in literature. Though animal data suggests the possibility of liver damage after chronic Khat exposure, literature reporting chronic Khat abuse as a causal factor for chronic end stage liver disease in humans is sparse. We would like to report a case of end stage chronic liver disease related to chronic Khat consumption and the outcome after liver transplantation.
\end{abstract}

Keywords: Khat; QAT; KAT; Chronic liver disease; Liver transplantation

\section{Introduction}

Chronic occult toxin exposure causing liver injury, fibrosis and leading to end stage cirrhosis can be easily missed especially in immigrant populations due to a lack of awareness of non-native rituals and practices of these communities in the local medical community. Khat leaf chewing is one such practice amongst East African and Yemenese men living in the UK that is known to be associated with significant medical adverse effects [1]. Liver disease in this group of people has been thought to have "cryptogenic" origin; others have had their liver disease attributed to other more preva-

Manuscript accepted for publication March 15, 2011

${ }^{a}$ Liver unit, Freeman Hospital, Freeman Road, Newcastle upon Tyne, NE7 7DN, UK

${ }^{\mathrm{b}}$ Institute of Cellular Medicine, Newcastle University, Framlington Place, Newcastle upon Tyne, NE2 4HH, UK

${ }^{c}$ Corresponding author: Imran Patanwala,

Email: i.m.patanwala@ncl.ac.uk

doi:10.4021/jmc168w lent causes such as autoimmune liver disease due to their atypical presentation $[2,3]$. Though Khat chewing can cause acute liver dysfunction in man and [4] chronic liver injury in animals [5], there is limited evidence in literature relating chronic liver injury to regular Khat use [6]. We present here a case report of end stage liver cirrhosis that we attribute to Khat toxicity based on clinical, biochemical and pathological features and the outcome post liver transplantation (OLT).

\section{Case Report}

A 33-year-old Somalian man was referred to our liver unit with a history of lethargy and an unknown duration of jaundice. He had no significant past medical history. Past surgical history included a laparotomy 12 yrs ago for intra-abdominal shrapnel related war injuries and previous, successful, treatment for chronic osteomyelitis. He had never been investigated or treated for liver disease in Somalia and denied IV drug abuse, alcohol consumption, using herbal remedies or being exposed to poisons. He had no tattoos. Family history was obscured by poor recall. He smoked $5 \mathrm{cpd}$. On examination he had a BMI of 31, signs of established chronic liver disease and abdominal scars from previous surgery. His blood test results were atypical in that his alkaline phosphatase (ALP) levels were disproportionately high and we detected unusual liver directed autoimmune activity with positive anti smooth muscle antibody titre $(1: 40)$. His Hepatitis B and Delta serology (Hepatitis B surface antigen positive, Hepatitis B core antibody positive, Hepatitis B e Antibody positive, Hepatitis Delta IgM negative and Hepatitis Delta IgG reactive) suggested chronic hepatitis B and Delta coinfection without detectable Hepatitis B viral DNA or Hepatitis Delta RNA. Imaging and endoscopy confirmed the presence of cirrhotic portal hypertension. Liver biopsy showed appearances of cirrhosis due to a chronic hepatitis.

He was offered follow up with treatment for portal hypertensive complications including diuretics and betablockers but rapidly developed debilitating hepatic encephalopathy with persistently raised blood ammonia levels despite therapy. He was listed and transplanted electively. Postoperatively, he developed hepatic artery thrombosis 


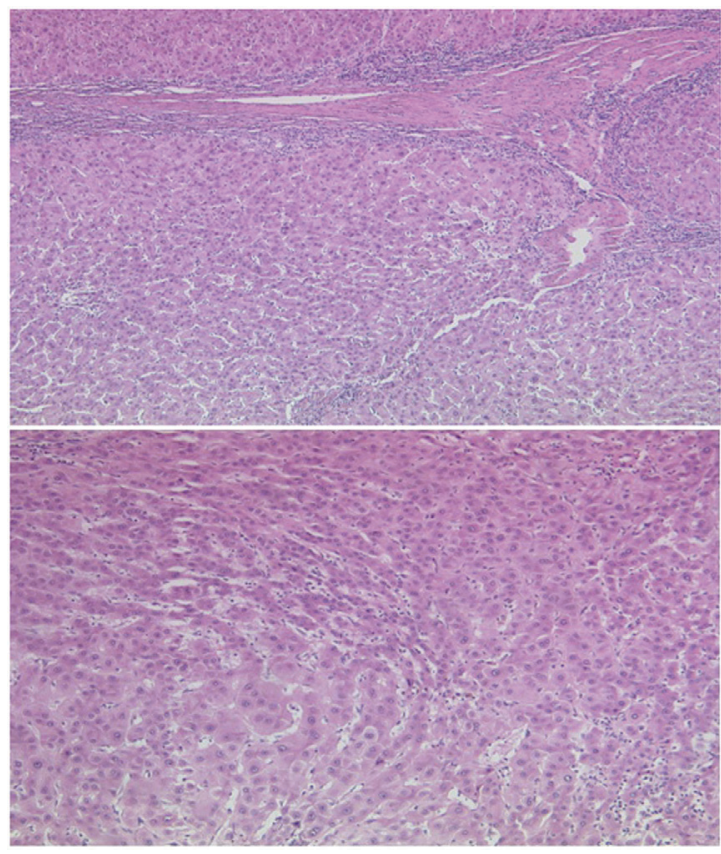

Figure 1. Explant liver histology (H\&E). Fibrous obliteration of portal vein radicles could be seen in many areas (upper). This was associated with nodular regenerative hyperplasia producing a nodule in nodule appearance within the background incomplete septal cirrhosis (lower).

within 10 days of transplantation and recurrent ventricular tachyarrhythmias. Pretransplant cardiac investigations were unremarkable. Explant histology review (Fig. 1) at this stage, showed incomplete septal cirrhosis and ongoing chronic hepatitis including interface hepatitis. However, areas of vascular occlusion involving hepatic and portal vein radicles and associated nodular regenerative hyperplasia leading to areas of 'nodule-in-nodule' formation were also present. These histological features suggested that the liver injury might have resulted from chronic exposure to a toxin. On further questioning him, he admitted to chewing large quantities of Khat on a daily basis. Unfortunately, over the next few weeks, he deteriorated secondary to sepsis and died from multi-organ failure.

\section{Discussion}

Khat is a green leafy plant grown in Yemen and East Africa. Its leaves are chewed for their euphoria inducing properties. It is estimated that almost $40 \%$ of Somalians in the UK chew Khat [7]. The principle ingredient in Khat is Cathinone, an alkaloid similar in properties to amphetamine. Adverse effects of Khat chewing include vascular thromboses in various territories [8] and cardiac toxicity [1]. Animals given cathinone develop high Alanine transaminase (ALT) and ALP levels, with ALP being more prominently raised than the ALT and liver histology revealing congestion in central liver veins and hepatocellular degeneration-regeneration activity. Longer term administration can result in inflammatory cell portal tract infiltration and porto-portal fibrosis [5]. Atypical autoimmune serology has been seen in Somalian men with liver disease in the UK [3] and interestingly, our patient demonstrated all of these above features.

Cessation of Khat consumption has been associated with recovery or stabilisation of liver disease in a majority of Somalian men with unexplained liver disease [3]. Currently, awareness and counselling against its use is the only treatment. Although the active ingredients of Khat are controlled substances in the UK under the Drugs Misuse Act of 1971, Khat itself is easily and freely available in the UK. It is banned in many European countries and in the USA. The free availability of Khat in the UK remains a subject of parliamentary debate. This case highlights the importance of increasing awareness of non-native rituals and practices in the local healthcare services in western communities that are managing an ever increasing immigrant population. It also establishes Khat as an etiological risk factor in chronic liver disease and suggests a potentiating effect of Khat toxicity on chronic hepatitis B and Delta virus mediated liver damage.

\section{Author Contributions}

Imran Patanwala participated in managing this case and wrote up the case report. Alastair Burt reviewed and reported histology, provided histology photograph for use in case report and its caption and, reviewed the report. Margaret F Bassendine participated in managing the case and reviewed the report. Mark Hudson managed the case in question and helped with reviewing and writing of the report.

\section{Conflicts of Interest}

None

\section{References}

1. Al-Habori M. The potential adverse effects of habitual use of Catha edulis (khat). Expert Opin Drug Saf 2005;4(6):1145-1154.

2. D'Souza R, Sinnott P, Glynn MJ, Sabin CA, Foster GR. An unusual form of autoimmune hepatitis in young Somalian men. Liver Int 2005;25(2):325-330.

3. McCune CA, Moorghan M, Gordon FH, CollinsPL. Liver disease and khat chewing in young Somalian men. Journal of Hepatology 2007:46(Supplement 1).

4. Brostoff JM, Plymen C, Birns J. Khat--a novel cause of drug-induced hepatitis. Eur J Intern Med 2006;17(5):383. 
5. Al-Mamary M, Al-Habori M, Al-Aghbari AM, Baker MM. Investigation into the toxicological effects of Catha edulis leaves: a short term study in animals. Phytother Res 2002;16(2):127-132.

6. Chapman MH, Kajihara M, Borges G, O’Beirne J, Patch D, Dhillon AP, Crozier A, et al. Severe, acute liver injury and khat leaves. N Engl J Med 2010;362(17):1642-
1644.

7. Advisory Council on the Misuse of Drugs, Khat(Qat): assessment of risk to the individual and communities in the UK. 2005.

8. de Ridder S, Eerens F, Hofstra L. Khat rings twice: Khat-induced thrombosis in two vascular territories. Neth Heart J 2007;15(7-8):269-270. 